Chronic Obstructive Pulmonary Diseases: Journal of the COPD Foundation

\author{
Original Research
}

\title{
Is Methicillin-Resistant Staphylococcus Aureus Colonization Associated with Worse Outcomes in COPD Hospitalizations?
}

\author{
Erin R. Narewski, DO ${ }^{1}$ Victor Kim, MD $^{1}$ Nathaniel Marchetti, DO ${ }^{1}$ Michael R. Jacobs, PharmD ${ }^{1}$ \\ Gerard J. Criner, MD $^{1}$
}

\begin{abstract}
Background: Although methicillin-resistant Staphylococcus aureus (MRSA) colonization is common in chronic obstructive pulmonary disease (COPD) patients, its effect on the course of COPD hospitalization remains unknown. Methods: Records of 160 patients hospitalized at our institution January 1, 2008 to May 1, 2010 with acute exacerbations of COPD who were screened for MRSA were examined and outcomes from their hospitalizations were quantified.

Results: Of the 160 patients, 33 (20.6\%) were MRSA colonized on screening. These patients had similar demographics, spirometry, Charlson Indexes, and APACHE-II scores when compared to patients who were not MRSA colonized ( $\mathrm{n}=127)$, but MRSA colonized patients had more hospitalizations within the 2 years prior to admission (2 [1-4.8] versus 1 [0-3], $p=0.03$ ). While hospitalized, MRSA colonized patients had a longer length of stay (9 [5.3-15.5] versus 5 [3-7.8] days, $p=0.01$ ) and more antibiotic days (7 [5-10.8] versus 5 [0-7] days, $p=0.01)$. They were also more likely to receive intensive care $(51.5 \%$ versus $23.6 \%, p=0.01)$ and to develop respiratory failure that required noninvasive ventilation ( $56.3 \%$ versus $38.2 \%, p=0.05)$. Trends towards increased use of invasive mechanical ventilation and readmission within 30 days were also present.

Conclusions: COPD patients colonized with MRSA have longer hospitalizations, require longer courses of antibiotics, and are more likely to require intensive care.
\end{abstract}

\begin{abstract}
Abbreviations: methicillin-resistant Staphylococcus aureus, MRSA; chronic obstructive pulmonary disease, COPD; acute exacerbations of COPD, AECOPD; cystic fibrosis, CF; forced expiratory volume in 1 second, FEV $_{\mathbf{1}}$; International Classification of Diseases, Ninth Revision, ICD-9; systemic inflammatory response syndrome, SIRS; human immunodeficiency virus, HIV; Acute Physiology and Chronic Health Evaluation - II , APACHE-II; standard deviation, SD; MRSA colonization positive, MRSA+; negative for MRSA, MRSA -; noninvasive positive pressure ventilation, NIPPV; confidence interval, CI; partial pressure of arterial $\mathrm{CO}_{2}, \mathrm{PaCO}_{2}$; body mass index, BMI; diabetes mellitus, DM; long term care facility, LTCF; white blood cell, WBC; forced vital capacity, FVC; interquartile range, IQR; intensive care unit, ICU

Funding Support: None

Date of Acceptance: April 27, 2015

Citation: Narewski ER, Kim V, Marchetti N, Jacobs MR, Criner GJ. Is methicillin- resistant Staphylococcus aureus colonization associated with worse outcomes in COPD hospitalizations? J COPD F. 2015; 2(3): 252-258. doi: http://dx.doi.org/10.15326/jcopdf.2.3.2014.0147.
\end{abstract}

A preliminary investigation on this concept was presented as an abstract at the American Thoracic Society International Conference in Denver, Colorado in May, 2011.

1 Temple University Hospital, Division of Pulmonary and Critical Care Medicine, Philadelphia, Pennsylvania

\section{Address correspondence to:}

Erin R Narewski, DO

Temple University Hospital, Division of Pulmonary and Critical

Care Medicine

3401 North Broad Street

Philadelphia, PA 19140

Erin.Narewski.DO@gmail.com 


\section{Keywords:}

methicillin-resistant Staphylococcus aureus colonization; chronic obstructive pulmonary disease; COPD; hospitalization outcomes

\section{Introduction}

Hospitals worldwide routinely screen patients for colonization with methicillin-resistant Staphylococcus aureus (MRSA). This has been prompted by the proliferation of colonization among hospitalized patients and as a method of preventing nosocomial transmission of this potentially virulent organism. ${ }^{1}$ Screening protocols and isolation of colonized patients have been found in some studies to be cost effective, as they prevent the development of nosocomial infections. ${ }^{2}$ Additionally, the cost of surgical site, vascular catheter, and urinary catheter-associated infections with this and other health care-associated organisms is no longer reimbursed by public insurance in the United States. ${ }^{3}$

Lower airways colonization with bacterial pathogens has been shown to increase the incidence of acute exacerbations of COPD (AECOPD). ${ }^{4}$ AECOPDs are known to result in increased health care utilization, hospitalization, mortality, and poor health-related quality of life which can persist after resolution of exacerbation. ${ }^{5}$ To our knowledge no data exist on how colonization with MRSA affects patients with COPD, although adverse outcomes of colonization have been demonstrated in patients with cystic fibrosis (CF), including decline in forced expiratory volume in 1 second $\left(F E V_{1}\right)$, worse radiographic appearance, and increased use of antibiotics. $6,7,8$

We performed a retrospective cohort study of COPD patients hospitalized with an acute exacerbation who were screened for colonization with MRSA to determine the impact of MRSA colonization on outcomes. We hypothesized that MRSA colonization would increase length of hospital stay, antibiotic use, and need for ventilatory support and intensive care in hospitalized patients with COPD.

\section{Materials and Methods}

\section{Patient Population}

After approval by our institutional review board, charts of consecutive patient visits coded for COPD were identified using a search of hospital records at our institution for International Classification of Diseases, Ninth Revision (ICD-9) Codes 490-496. Individuals were 40-99 years of age and admitted between January 1, 2008 and May 1, 2010 for AECOPD.

Admission criteria were determined by chart review. All patients had a physician's diagnosis of AECOPD. Criteria for the diagnosis of COPD included $\geq 10$ pack years of smoking, a documented physical examination and medication list consistent with COPD, and a chest radiograph without findings suggesting an alternative diagnosis. Criteria for AECOPD included the presence of at least 1 documented symptom including increased dyspnea or cough and increased or changed sputum production.

Patients with a concomitant diagnosis of pneumonia, bacterial tracheobronchitis or rhinosinusitis, or systemic inflammatory response syndrome (SIRS) were excluded. Additional exclusion criteria included absence of an MRSA screening test, current cancer diagnosis, dialysis or serum creatinine $>2.5 \mathrm{mg} / \mathrm{dL}$, human immunodeficiency virus (HIV) infection, prior organ transplant, and hospice or comfort care status during the hospitalization. These were selected to reduce factors that influence hospitalization outcomes. See Figure 1.

\section{Determination of MRSA Colonization}

All MRSA screening tests were performed by microbial culture. BBL $^{\mathrm{TM}}$ CultureSwabs ${ }^{\mathrm{TM}}$ were used to collect the sample from the nares, or Lukens Tubes / sterile collection cups for sputum samples within 2 hours of admission. These samples were then plated onto $\mathrm{BBL}^{\mathrm{TM}}$ CHROMagar ${ }^{\mathrm{TM}}$ MRSA II Plates and incubated for 24 hours at $35-37^{\circ} \mathrm{C}$ in a dark environment free of carbon dioxide. Colonies suspected to be MRSA were then confirmed using Patorex ${ }^{\mathrm{TM}}$ Staph Plus agglutination tests. If no colonies were found the plate was incubated for an additional 24 hours before confirming the absence of MRSA. Patients were considered MRSA colonized if the screening nasopharyngeal swab or sputum sample contained MRSA but did not meet clinical criteria for infection as described above.

Some patients underwent screening using both sputum sample and nasopharyngeal swab. Only if both modalities revealed positive results was the patient deemed colonized with MRSA. No patient with discordant sputum and nasopharyngeal swab results was included in this study. All patients with positive screening tests for MRSA colonization were placed on contact isolation. 


\section{Figure 1. A Breakdown of Patient Charts Examined}

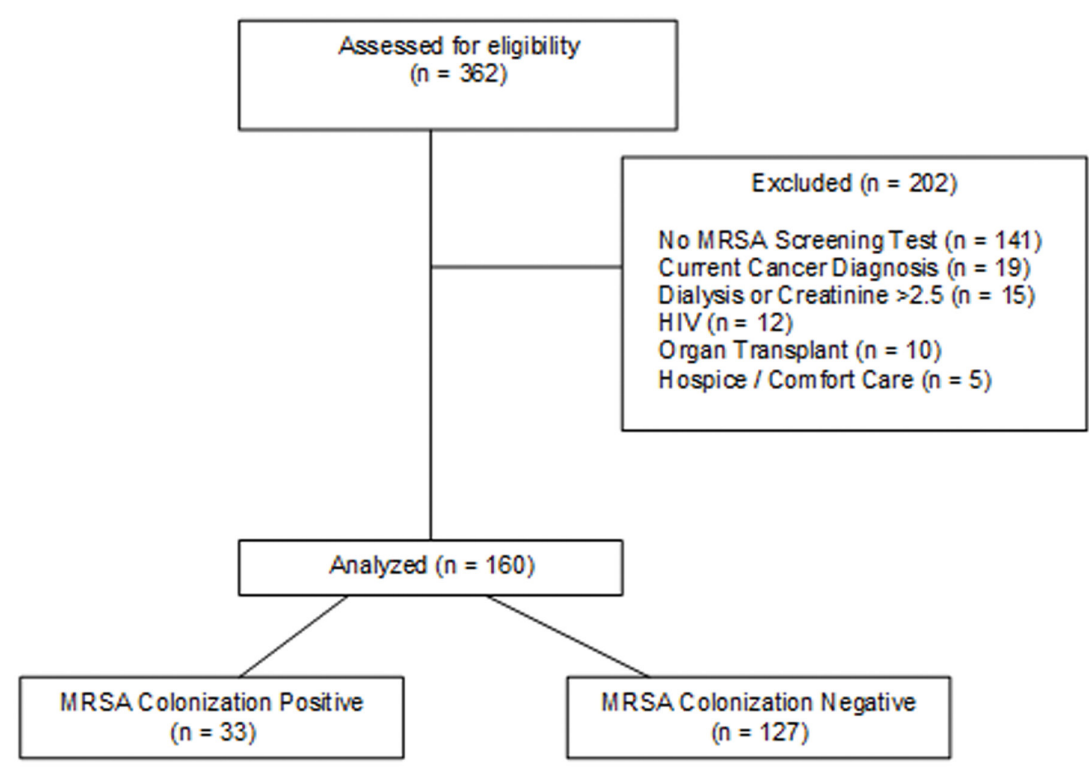

methicillin -resistant Staphylococcus aureus: MRSA; human immunodeficiency virus: HIV invasive or noninvasive ventilation, and need for intensive care. Hospitalizations at other institutions were not identified.

Data regarding the severity of disease were also collected. An Acute Physiology and Chronic Health Evaluation - II (APACHE-II) score and a Charlson Comorbidity Index was calculated for each patient at the time of admission. The radiologist's reports of admission chest radiographs were also evaluated for evidence of underlying parenchymal lung disease. Finally, to account for other variables in severity of illness and health status, we quantified the number of additional medical diagnoses noted in each patient's chart.

Treatment failure was the primary outcome, and represented a composite variable of need for ventilation (either invasive or non-invasive) or need for intensive care. Additionally, we collected data on length of stay, days of antibiotic treatment, 30 day readmission rates, and inpatient mortality.

\section{Data Collection}

Data from the hospitalization selected using the criteria above, and prior hospitalizations when present, were collected by chart review by the investigator (E.N.), who was blinded to MRSA colonization screening test results. When available, spirometry results were obtained using an electronic database of studies performed at our institution within 1 year prior to the index hospitalization. All spirometry was performed according to American Thoracic Society / European Respiratory Society standards. ${ }^{9}$

Data on risk factors associated with MRSA colonization were also collected. These include socioeconomic status, prior history of hospitalization, residence in a long-term care facility, and diabetes mellitus. ${ }^{10}$ In order to estimate socioeconomic status, we collected the postal code of each patient and used demographic data from the 2000 U.S. Census for the area to estimate median local household income. Postal codes with an average annual household income $<30,000$ U.S. dollars were considered low income. We evaluated the effect of prior hospitalization by analyzing the prevalence of hospitalizations within 1 year prior to the index hospitalization. We then evaluated all most recent prior hospitalizations for specific risk factors for colonization with MRSA, including length of stay, antibiotic use,

\section{Data Analysis}

Data were analyzed using JMP-10 / SAS (Cary, North Carolina). Pearson's Chi-squared test was used for nominal variables. Unpaired t-test was used for continuous variables. Non-parametric data were analyzed using the Wilcoxon rank-sum test. All data are presented as percentage, mean \pm standard deviation (SD), or median and interquartile range where indicated. $P$-values $<0.05$ were considered statistically significant.

\section{Results}

A total of 362 patient charts were examined. Of these, 202 patients were excluded, mostly due to no MRSA screening test performed, as demonstrated in Figure 1. Of the 160 patients meeting criteria for our study, 33 (20.6\%) were MRSA colonization positive (MRSA +).

Demographic data for MRSA + patients were similar to patients screening negative for MRSA (MRSA -). Table 1 includes these results. Data on previous hospitalizations, which were more frequent in MRSA + patients, are also presented in Table 1. Table 1 also compares patients using their local estimated household income. There was no significant difference in MRSA prevalence between patients living in low-income areas and nonlow-income areas. The number of comorbid diagnoses 


\section{Table 1. Demographic Data, MRSA Risk Factors, and Physiology}

\begin{tabular}{|c|c|c|c|}
\hline$n$ & $\begin{array}{c}\text { MRSA + } \\
33\end{array}$ & $\begin{array}{l}\text { MRSA - } \\
127\end{array}$ & $\boldsymbol{P}$ \\
\hline Age (years) & $63.0 \pm 12.4$ & $64.1 \pm 10.4$ & 0.60 \\
\hline Male Gender & $16(48.5)$ & $50(40.9)$ & 0.28 \\
\hline Caucasian & $14(42.4)$ & 38 (29.9) & \\
\hline African American & $14(42.4)$ & $78(61.4)$ & 0.13 \\
\hline Hispanic & $5(15.2)$ & $11(8.7)$ & \\
\hline Current Smokers & $8(24.2)$ & $38(29.9)$ & 0.80 \\
\hline $\mathrm{BMI}\left(\mathrm{kg} / \mathrm{m}^{2}\right)$ & $32.0 \pm 10.4$ & $30.8 \pm 9.9$ & 0.54 \\
\hline $\begin{array}{l}\text { Hospitalizations within } \\
2 \text { Years Prior to Admission }\end{array}$ & $2(1-4.8)$ & $1(0-3)$ & 0.03 \\
\hline $\begin{array}{l}\text { Residence in Low Income } \\
\text { Postal Code }\end{array}$ & $23(69.7)$ & $79(62.2)$ & 1 \\
\hline $\begin{array}{l}\text { Number of Additional } \\
\text { Diagnoses }\end{array}$ & $3.7 \pm 1.5$ & $3.3 \pm 1.6$ & 0.21 \\
\hline DM Diagnosis & $12(36.4)$ & $28(22.1)$ & 0.07 \\
\hline Arrival from a LTCF & $1(3.0)$ & $3(2.4)$ & 0.61 \\
\hline $\begin{array}{l}\text { Radiographic Abnormalities } \\
\text { Other Than Emphysema }\end{array}$ & $10(30.3)$ & $34(26.8)$ & 0.42 \\
\hline $\mathrm{WBC}\left(\mathrm{k} / \mathrm{mm}^{3}\right)$ & $11.9 \pm 4.8$ & $10.6 \pm 5.0$ & 0.16 \\
\hline Charlson Index & $4.39 \pm 2.2$ & $4.25 \pm 1.8$ & 0.70 \\
\hline APACHE-II Score & $11.18 \pm 4.7$ & $11.01 \pm 4.5$ & 0.84 \\
\hline $\mathrm{PCO}_{2}$ Data & $\mathbf{n}=\mathbf{3 2}$ & $\mathbf{n}=116$ & \\
\hline Admission $\mathrm{PaCO}_{2}(\mathrm{~mm} \mathrm{Hg})$ & $55.6 \pm 16.2$ & $55.7 \pm 20.7$ & 0.99 \\
\hline Spirometry & $\mathrm{n}=16$ & $n=46$ & \\
\hline FVC (\%) & $63.06 \pm 15.3$ & $64.04 \pm 16.2$ & 0.83 \\
\hline $\mathrm{FEV}_{1}(\%)$ & $33.13 \pm 12.5$ & $40.37 \pm 18.2$ & 0.15 \\
\hline $\mathrm{FEV}_{1} / \mathrm{FVC}$ & $41.43 \pm 13.6$ & $49.11 \pm 19.5$ & 0.15 \\
\hline
\end{tabular}

Data are presented as $\mathrm{n}(\%)$ or mean $\pm \mathrm{SD}$

methicillin-resistant Staphylococcus aureus: MRSA; positive: +, negative: -; $P$-Value: $P$; body mass index; $\mathrm{BMI}$; diabetes mellitus:DM; long term care facility:LTCF; white blood cell: WBC; partial pressure of arterial $\mathrm{CO}_{2}$ : $\mathrm{PaCO}_{2}$; forced vital capacity: FVC; forced expiratory volume in 1 second: $\mathrm{FEV}_{1}$

present per patient, the diagnosis of diabetes mellitus, and prior residence in a long-term care facility did not differ significantly between groups. No differences were noted in severity of lung disease as measured by spirometry, when available. White blood cell counts and the prevalence of underlying parenchymal lung disease did not differ between groups. Severity of illness, as quantified by the Charlson Index and APACHE-II Score, was also not different between groups.

Table 2 shows the analysis of the most recent prior hospitalizations, when present. During prior hospitalizations, MRSA + patients had longer lengths of stay and were at any time in their hospitalization more likely to require noninvasive positive pressure ventilation (NIPPV).

\section{Table 2. Prevalence of Risk Factors During Most Recent Prior Hospitalization}

\begin{tabular}{lrrl} 
& \multicolumn{1}{c}{ MRSA + } & \multicolumn{1}{c}{ MRSA - } & P \\
\multicolumn{1}{c|}{$\mathrm{n}$} & \multicolumn{1}{c}{21} & \multicolumn{1}{c}{51} & \\
\hline Antibiotics Used & $11(52.4)$ & $27(52.9)$ & 0.62 \\
\hline Antibiotic Days, median & $1(0-10)$ & $1(0-7)$ & 0.58 \\
\hline ICU Admission & $4(19.1)$ & $8(15.7)$ & 0.48 \\
\hline $\begin{array}{l}\text { Invasive Mechanical } \\
\text { Ventilation }\end{array}$ & $2(9.5)$ & $5(9.8)$ & 0.66 \\
\hline NIPPV & $9(42.9)$ & $8(15.7)$ & 0.02 \\
\hline AECOPD During Stay & $11(52.4)$ & $28(54.9)$ & 0.68 \\
\hline Length of Stay, median & $7(4.5-10)$ & $3(2-6)$ & 0.01 \\
\hline
\end{tabular}

Data are presented as $\mathrm{n}(\%)$ or mean (IQR).

methicillin-resistant Staphylococcus aureus: MRSA; positive: +, negative: -; $P$-Value: $P$; interquartile range: IQR; intensive care unit: ICU; NIPPV: non-invasive positive airway pressure; acute exacerbation of chronic obstructive pulmonary disease: AECOPD;

MRSA + patients were more likely to have respiratory failure (NIPPV or invasive ventilation) or to require intensive care. Figure 2 illustrates the significant differences in the primary outcome between groups. A composite variable of these was our primary outcome. The odds ratio for the primary outcome in MRSA + patients was 2.61 (95\% confidence interval [CI] 1.165.87). Table 3 reveals that MRSA + patients stayed longer in the hospital (9 [5.3-15.5] versus 5 [3-7.8] days, $p=0.01$ ), had a longer duration of treatment with antibiotics (7 [5-10.8] versus 5 [0-7] days, $p=0.01)$, were more likely to receive intensive care $(51.5 \%$ versus $23.6 \%, p=0.01$ ) and developed respiratory failure that required NIPPV (56.3\% versus $38.2 \%, p=0.05$ ). Trends towards an increased need for invasive mechanical ventilation and readmission within 30 days were present but were not statistically significant. In-hospital mortality ( $3.0 \%$ versus $0.8 \%, p=0.37$ ) was not different between groups.

\section{Discussion}

Patients colonized with MRSA, as determined by 


\section{Figure 2. Primary Outcome: Treatment Failure}

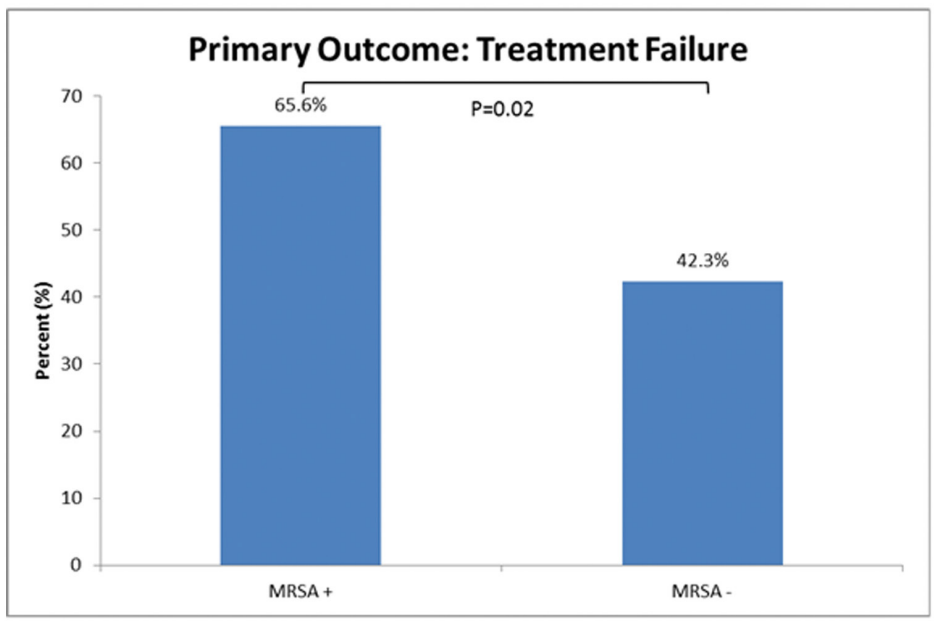

methicillin-resistant Staphylococcus aureus: MRSA; positive : +; negative: -; $P$-value: $P$

\section{Table 3. Outcomes}

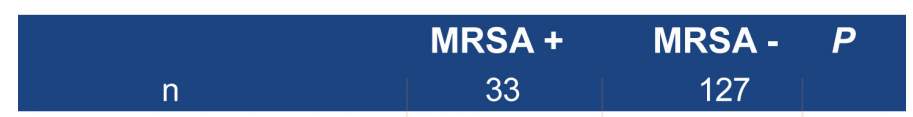

\begin{tabular}{|c|c|c|c|}
\hline Length of Stay & $9(5.3-15.5)$ & $5(3-7.8)$ & 0.01 \\
\hline Antibiotic Days & $7(5-10.8)$ & $5(0-7)$ & 0.01 \\
\hline Transferred to ICU & $17(51.5)$ & $30(23.6)$ & 0.01 \\
\hline Any Ventilatory Support & $20(60.6)$ & $47(38.2)$ & 0.01 \\
\hline NIPPV & $18(56.3)$ & $47(38.2)$ & 0.05 \\
\hline $\begin{array}{l}\text { Invasive Mechanical } \\
\text { Ventilation }\end{array}$ & 7 (21.9) & $18(14.6)$ & 0.23 \\
\hline Expired & $1(3.0)$ & $1(0.8)$ & 0.37 \\
\hline Readmitted within 30 days & $6(18.2)$ & $18(14.2)$ & 0.37 \\
\hline
\end{tabular}

Data are presented as $\mathrm{n}(\%)$ or mean (IQR).

methicillin-resistant Staphylococcus aureus: MRSA; positive: +, negative: -; $P$-Value: $P$; interquartile range: IQR; intensive care unit: ICU; NIPPV: non-invasive positive airway pressure ventilation

nasopharyngeal swab or by sputum culture, have similar demographic characteristics with MRSA-patients. However, they require longer lengths of stay and more antibiotics when hospitalized for AECOPD. They are also more likely to fail treatment and require NIPPV or intensive care. These findings are strengthened by the collection of additional data, including the prevalence of risk factors for MRSA, spirometry, and indexes of disease severity. Given that MRSA colonization is known to be more common in patients with poorer socioeconomic status, diabetes mellitus, and frequent hospitalization, ${ }^{10}$ data on these risk factors were analyzed in addition to standard demographic data. APACHE-II scores and Charlson indexes were also collected to estimate the severity of disease, due to concern that patients colonized with MRSA would have a greater severity of illness at baseline. Spirometry, chest radiographs, admission white blood cell count, and partial pressure of arterial $\mathrm{CO}_{2}\left(\mathrm{PaCO}_{2}\right)$ were similarly not different between groups, suggesting that MRSA colonization is a unique variable, independent of disease severity, which predicts worse outcomes in patients with COPD.

These data illustrate poor outcomes during hospitalizations in patients admitted with AECOPD who are colonized with MRSA. This supports the hypothesis that MRSA may be a harmful colonizer in patients with COPD and corroborates previously published data on the detrimental effects of airways colonization with other organisms. ${ }^{4}$ Research has revealed that some bacteria evoke a greater pulmonary inflammatory response than others. ${ }^{11}$ MRSA in particular has been studied thoroughly in its role as a colonizer and inflammatory pathogen. It is known to take advantage of multiple cell-surface receptors to invade the pulmonary parenchyma, ${ }^{12}$ and once there to interfere with host B-cell immune response ${ }^{13}$ and cause granulocyte lysis. ${ }^{14}$ It secretes a variety of virulence factors which differ by local isolate, but all of these stimulate host inflammation. ${ }^{14,15}$

Sethi et al have explored the role of infectious organisms both in stable COPD and in acute exacerbations. ${ }^{16}$ Approximately $50 \%$ of AECOPDs are related to colonization with a new microorganism. In particular, H. influenzae, Moraxella catarrhalis, Streptococcus pneumoniae, and Pseudomonas aeruginosa are related to exacerbations. Stable COPD patients are also colonized with pathogens $25 \%-50 \%$ of the time. ${ }^{16}$

Much of the research on the clinical consequences of MRSA colonization has focused on patients with CF. Cystic fibrosis patients experience a more rapid rate of decline in $\mathrm{FEV}_{1}$ when colonized with MRSA, which suggests that MRSA colonization may have similar effects on COPD patients. ${ }^{6}$ Moreover, patients with CF who are colonized with MRSA require more courses of intravenous antibiotics and have a worse radiographic appearance than CF patients who are not colonized. ${ }^{7}$ This supports the hypothesis that MRSA is likely to be associated with worse outcomes when COPD patients become colonized. However, the prevalence of MRSA 
colonization in populations of CF patients is reported to be low. ${ }^{7,8}$ In contrast, most patients with COPD have many risk factors for MRSA colonization including prior hospitalizations and antibiotic use, ${ }^{17}$ and many patients are colonized with MRSA. ${ }^{18}$ Outcomes in surgical, dialysis, intensive care unit, and transplant patients colonized with MRSA are also worse, primarily due to increased invasive MRSA infections. 19,20,21,22,23

Although this study illustrates an association between MRSA colonization and complex hospitalizations in patients with AECOPD, some limitations are worthy of mention. The patients enrolled were from a single institution and may not represent the entire COPD population, and patients were selected based on whether testing was performed. In addition, the study did not differentiate between patients with a single positive screening test for MRSA and persistently positive patients. Also, the time of colonization and duration of colonization is not known in these patients. Although efforts were made to determine if patients colonized with MRSA were more ill than patients not colonized, colonization may still be a marker of patients with more severe disease, rather than a contributor to the pathogenesis of COPD and AECOPD. This study did not address the question of whether decolonization may impact outcomes. Prior research has revealed that decolonization resulted in decreased transmission of MRSA. $^{24}$ In our study, the rate of MRSA colonization among screened patients was high. We believe this to be related to selection bias in those screened, as those meeting criteria for screening had more risk factors for MRSA than the general population of patients being admitted for AECOPD. Therefore, findings from this population may not be broadly applicable to other institutions or to other patients with COPD. Finally, this study examined only short-term outcomes in hospitalized COPD patients colonized with MRSA. The effect of colonization on lung function and quality of life and the effect of colonization on stable COPD patients have yet to be determined. Prospective studies are needed to further explore these questions.

Despite these limitations, this study found that patients hospitalized for AECOPD, when colonized with MRSA, required more antibiotics and a longer length of hospital stay. They were also more likely to require intensive care and non-invasive ventilation. These patients had a more significant history of prior hospitalization despite having a similar severity of disease to patients not colonized with MRSA. Positive MRSA screening tests may be associated with poor outcomes in hospitalized patients with AECOPD. Larger studies are needed to corroborate these findings.

\section{Acknowledgements}

Author contributions: Erin R. Narewski, DO - principal investigator / corresponding author / guarantor; Victor Kim, MD - contributing author; Nathaniel Marchetti, DO- contributing author; Gerard J. Criner, MDcontributing author; Michael Jacobs, PharmD -advice and guidance.

\section{Declaration of Interest}

Erin R. Narewski has no conflicts of interest. Victor Kim has nothing to disclose in relationship to this manuscript but has served on an advisory committee for CSA and has participated in clinical trials sponsored by Boehringer Ingelheim, Glaxo-Smith-Kline, and Roche pharmaceuticals. He is supported by NHLBI K23HL094696. Nathaniel Marchetti has no conflicts of interest. Michael Jacobs has no conflicts of interest Gerard J. Criner has received research grants from: Boehringer Ingelheim, Forest, Actelion, Glaxo-SmithKline, AstraZeneca, Pearl, Novartis Pharmaceuticals, Pulmonx, PneumRX and Aeris Therapeutics. All research grant monies are deposited and controlled by Temple University. 


\section{References}

1. Boyce JM. Should we vigorously try to contain and control methicillin resistant Staphylococcus aureus? Infect Control Hosp Epidemiol. 1991;12(1):46-54.

doi: http://dx.doi.org/10.1086/646237

2. Papia G, Louie M, Tralla A, et al. Screening high risk patients for methicillin resistant Staphylococcus aureus on admission to the Hospital: is it Cost Effective? Infect Control Hosp Epidemiol. 1999; 20:473-477. doi: http://dx.doi.org/10.1086/501655

3. Stone, P. Changes in Medicare reimbursement for hospitalacquired conditions including infections. Am J of Infect Control. 2009; 37(9):A17-A18. doi: http://dx.doi.org/10.1016/j.ajic.2009.07.001

4. Patel IS, Seemungal TAR, Wilks M, Lloyd-Owen SJ, Donaldson GC, Wedzicha JA. Relationship between bacterial colonization and the frequency, character, and severity of COPD exacerbations. Thorax. 2002;57(9):759-764. doi: http://dx.doi.org/10.1136/thorax.57.9.759

5. Celli BR, Barnes PJ. Exacerbations of chronic obstructive pulmonary disease. Eur Respir J. 2007;29(6):1224-1238. doi: http://dx.doi.org/10.1183/09031936.00109906

6. Dasenbrook EC, Merlo CA, Diener-West M, Lechtzin N, Boyle, MP. Persistent methicillin-resistant Staphylococcus aureus and rate of FEV1 decline in cystic fibrosis. Am J Respir Crit Care Med. 2008;178(8):814-821. doi: http://dx.doi.org/10.1164/rccm.200802-3270C

7. Miall LS, McGinley NT, Brownlee KG, Conway SP. Methicillin resistant Staphylococcus aureus (MRSA) infection in cystic fibrosis. Arch Dis Child. 2001; 84(2): 160-162. doi: http://dx.doi.org/10.1136/adc.84.2.160

8. Boxerbaum B, Jacobs MR, Cechner RL. Prevalence and significance of methicillin-resistant Staphylococcus aureus in patients with cystic fibrosis. Pediatr Pulmonol. 1988; 4(3):159163. doi: http://dx.doi.org/10.1002/ppul.1950040307

9. Miller MR, Hankinson J, Brusasco V, et al. ATS/ERS task force: standardization of lung function testing, standardization of spirometry. Eur Respir J. 2005; 26(2):319-338. doi: http://dx.doi.org/10.1183/09031936.05.00034805

10. Forster A, Oake N, Roth V, et al. Patient-level factors associated with methicillin-resistant Staphylococcus aureus carriage at hospital admission: A systemic review. Am J of Infect Control. 2013;41(3):214-220.

doi: http://dx.doi.org/10.1016/j.ajic.2012.03.026

11. Marin A, Monso E, Garcia-Nunez N, et al. Variability and effects of bronchial colonization in patients with moderate COPD. Eur Respir J. 2010;35(2):295-302.

doi: http://dx.doi.org/10.1183/09031936.00126808

12. Soong G, Martin F, Chun J, Cohen TS, Ann DS, Prince A. Staphylococcus aureus protein A mediates invasion across airway epithelial cells through activation of RhoA GTPase signaling and proteolytic activity. J Biol Chem. 2011; 286(41):35891-35898. doi: http://dx.doi.org/10.1074/jbc.M111.295386

13. Kim HK, Kim HY, Schneewind O, Missiakas D. Identifying protective antigens of Staphylococcus aureus, a pathogen that suppresses host immune responses. The FASEB Journal. 2011; 25(10):3605-3612. doi: http://dx.doi.org/10.1096/fj.11-187963
14. Below S, Konkel A, Zeeck C, et al. Virulence factors of Staphylococcus aureus induce Erk-MAP kinase activation and c-Fos expression in S9 and 16HBE14o- human airway epithelial cells. Am J Physiol Lung Cell Mol Physiol. 2009; 296(3):L470-L479. doi: http://dx.doi.org/10.1152/ajplung.90498.2008

15. Schlievert P. Cytolysins, superantigens, and pneumonia due to community-associated methicillin-resistant Staphylococcus aureus. J Infect Dis. 2009;200(5):676-678. doi: http://dx.doi.org/10.1086/605333

16. Sethi S, Murphy T. Infection in the pathogenesis and course of chronic obstructive pulmonary disease. New Eng J Med. 2008; 359(22):2355-2365. doi: http://dx.doi.org/10.1056/NEJMra0800353

17. Hidron A, Kourbatova E, Halvosa JS, et al. Risk factors for colonization with methicillin-resistant Staphylococcus aureus (MRSA) in patients admitted to an urban hospital: emergence of community-associated MRSA nasal carriage. Clin Infect Dis. 2005; 41(2):159-166. doi: http://dx.doi.org/10.1086/430910

18. Gorwitz R, Kruszon-Moran D, McAllister S, et al. Changes in the prevalence of nasal colonization with Staphylococcus aureus in the United States 2001-2004. J Infect Dis. 2007;197(9):1226-1234. doi: http://dx.doi.org/10.1086/533494

19. Von Eiff C, Becker K, Machka K, Stammer H, Peters G. Nasal carriage as a source of Staphylococcus aureus bacteremia. $N$ Engl J Med. 2001; 344(1):11-16. doi: http://dx.doi.org/10.1056/NEJM200101043440102

20. Weinstein H. The relation between the nasal-staphylococcalcarrier state and the incidence of postoperative complications. $N$ Engl J Med. 1959; 260:1303-1308. doi: http://dx.doi.org/10.1056/NEJM195906252602601

21. Safdar N, Bradley E. The risk of infection after nasal colonization with Staphylococcus aureus. Am J Med. 2008;121(4):310-315. doi: http://dx.doi.org/10.1016/j.amjmed.2007.07.034

22. Garrouste-Orgeas M, Timsit JF, Kallel H, et al. Colonization with methicillin-resistant Staphylococcus aureus in ICU patients: morbidity, mortality, and glyopeptide use. Infect Control Hosp Epidemiol. 2001; 22(11):687-692. doi: http://dx.doi.org/10.1086/501846

23. Russel DL, Flood A, Xaroda TE, et al. Outcomes of colonization with MRSA and VRE among liver transplant candidates and recipients. Am J Transplant. 2008; 8(8):1737-1743. doi: http://dx.doi.org/10.1111/j.1600-6143.2008.02304.x

24. Huang SS, Septimus E, Kleinman K, et al. Targeted verses universal decolonization to prevent ICU infection. New Eng J of Med. 2013; 368:2255-2265. doi: http://dx.doi.org/10.1056/NEJMoa1207290 